\title{
INTUITIONISTIC FUZZY EDAS METHOD: AN APPLICATION TO SOLID WASTE DISPOSAL SITE SELECTION
}

\author{
Cengiz KAHRAMAN ${ }^{a}$, Mehdi KESHAVARZ GHORABAEE ${ }^{\mathrm{b}}$, Edmundas Kazimieras ZAVADSKAS', \\ Sezi CEVIK ONAR ${ }^{\text {a, }}$ Morteza YAZDANI ${ }^{\text {, }}$, Basar OZTAYSI ${ }^{a}$ \\ ${ }^{a}$ Department of Industrial Engineering, Istanbul Technical University, 34367 Macka, Istanbul, Turkey \\ ${ }^{b}$ Department of Industrial Management, Faculty of Management and Accounting, \\ Allameh Tabataba’i University, Tehran, Iran \\ ' Department of Construction Technology and Management, Faculty of Civil Engineering, \\ Vilnius Gediminas Technical University, Lithuania \\ ${ }^{d}$ Department of Management Science, Universidad Europea de Madrid, Madrid, Spain
}

Submitted 21 Sep. 2016; accepted 09 Jan. 2017

\begin{abstract}
Evaluation based on Distance from Average Solution (EDAS) is a new multicriteria decision making (MCDM) method, which is based on the distances of alternatives from the average scores of attributes. Classical EDAS has been already extended by using ordinary fuzzy sets in case of vague and incomplete data. In this paper, we propose an interval-valued intuitionistic fuzzy EDAS method, which is based on the data belonging to membership, nonmembership, and hesitance degrees. A sensitivity analysis is also given to show how robust decisions are obtained through the proposed intuitionistic fuzzy EDAS. The proposed intuitionistic fuzzy EDAS method is applied to the evaluation of solid waste disposal site selection alternatives. The comparative and sensitivity analyses are also included.
\end{abstract}

Keywords: EDAS, ordinary fuzzy sets, intuitionistic fuzzy sets, solid waste, site selection, average solution, multicriteria decision making (MCDM).

\section{Introduction}

EDAS method uses average solution for appraising the alternatives. It was developed by Keshavarz Ghorabaee et al. (2015). In this method, two measures which is called PDA (positive distance from average) and NDA (negative distance from average) are considered for the appraisal. This method is very useful when we have some conflicting criteria. In the compromise MCDM methods such as VIKOR and TOPSIS, the best alternative is obtained by calculating the distance from positive and negative ideal solutions. The best alternative has least distance from positive ideal solution (PIS) and largest distance from negative ideal solution (NIS) in these MCDM methods. In EDAS method, the best alternative is selected with respect to the distances from average solution: The positive distance from average (PDA) and the negative distance from average (NDA). An alternative having higher values of PDA and lower values of NDA is better (Keshavarz Ghorabaee et al. 2015).
The fuzzy set theory is a powerful tool in order to overcome ambiguous information. It was developed by Zadeh (1965) and has been used in various areas including multicriteria decision making (Liu et al. 2012), aggregation operations (Liu et al. 2016; Liu, Jin 2012; Liu, P. D., Liu, Y. 2014), definition of uncertain linguistic variables (Liu et al. 2011; Liu, Yu 2014), etc. EDAS has been extended to ordinary fuzzy EDAS by Keshavarz Ghorabaee et al. (2016) to handle the MCDM problems under fuzzy environment. Fuzzy sets have a history starting from ordinary fuzzy sets and extending to other types of fuzzy sets as illustrated in Figure 1 (Kahraman et al. 2015, 2016).

Intuitionistic fuzzy sets (IFS) have been proposed by Atanassov (1986). IFSs are very effective to deal with uncertainty and vagueness. Therefore, the decision makers can utilize intuitionistic fuzzy sets, especially the interval-valued intuitionistic fuzzy sets (IVIFS) introduced by Atanassov and Gargov (1989) to better express the information of the candidates under incomplete and

\footnotetext{
Corresponding author: Cengiz Kahraman

E-mail:kahramanc@itu.edu.tr
} 
uncertain information environment. IFS can consider the membership and nonmembership degrees of the elements of a set at the same time, which their sum is not necessarily equal to one. Multicriteria decision making with interval-valued intuitionistic fuzzy sets has received a great deal of attention from researchers recently (Cevik Onar et al. 2015; Oztaysi et al. 2015). Later, intuitionistic fuzzy sets have been considered as a special subset of neutrosophic sets by Smarandache (1998) and have been extended by some researchers (Liu, Shi 2015; Liu, Wang 2014).

It is evident from the previous studies in the literature, there has been no work extending EDAS to IVIF EDAS model to solve a multicriteria problem under fuzzy environment. The proposed method can be used to compare electronic waste or solid waste disposal technologies, disposal site alternatives, and hazardous material removal technologies for environmental protection.

To the best of our knowledge, this is the first paper extending EDAS method to IVIF EDAS. The performance of the proposed IVIF EDAS is illustrated through a solid waste disposal site selection problem. Solid waste is the unwanted or useless solid materials generated from combined residential, industrial and commercial activities in a given area. Management of solid waste reduces or eliminates adverse impacts on the environment and human health and supports economic development and improved quality of life. A number of processes are involved in effectively managing waste for a municipality. These include monitoring, collection, transport, processing, recycling and disposal.

The rest of this paper is organized as follows. Section 1 includes a literature review on EDAS method and gives the steps of classical EDAS. Section 2 presents the preliminaries of ordinary fuzzy sets and intuitionistic fuzzy sets together with the steps of ordinary fuzzy EDAS and intuitionistic fuzzy EDAS. Section 3 presents the

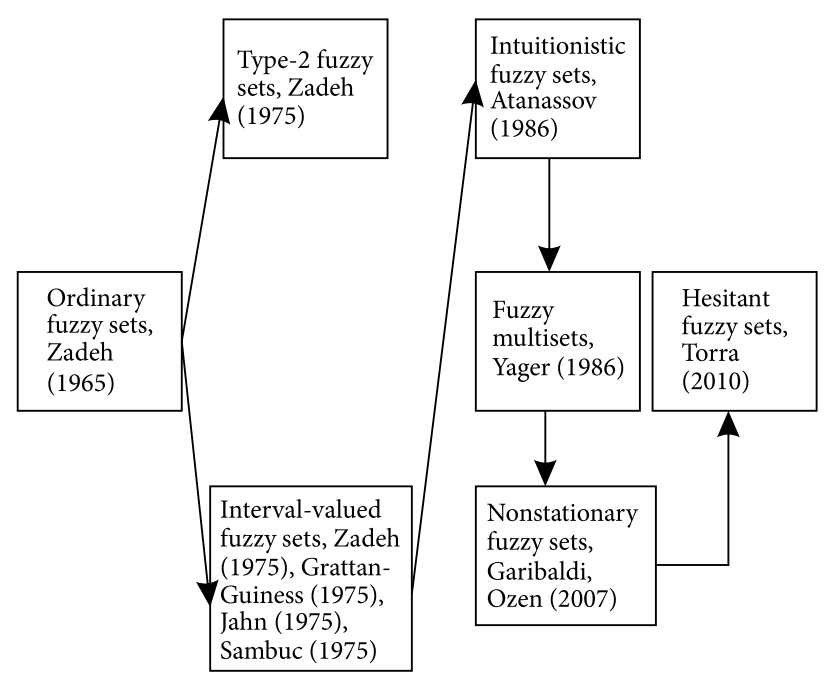

Fig. 1. Extensions of fuzzy sets applications of classical (crisp), ordinary fuzzy, and intuitionistic fuzzy EDAS for the solution of a solid waste disposal site selection problem. The last Section concludes the paper.

\section{EDAS method}

Curiosity of researchers to inventing new MCDM methods is getting competitive. Trends of MCDM methods development since initial days showed enormous interest among researchers in this area to build robust structures in order to handle complex decisions. Their precise effort was concentrated on realizing requirements of decision system, then implementing variables and parameters, and interconnecting those variables in a logical manner to establish specific tool. Many MCDM methods have been created and then extended. Analytical hierarchy process (Deng et al. 2014), ANP (Van Horenbeek, Pintelon 2014), TOPSIS (Büyüközkan, Çifçi 2012; Seçme et al. 2009), VIKOR (Mardani et al. 2016), ARAS (Turskis et al. 2013), COPRAS (Turanoglu Bekar et al. 2016), SAW (Hashemkhani Zolfani et al. 2012), ELECTRE (Figueira et al. 2013), PROMETHEE (Corrente et al. 2013), DEMATEL (Yeh, Huang 2014), MOORA (Stanujkic et al. 2014; Yazdani 2015), WASPAS (Chakraborty, Zavadskas 2014), SWARA (Kouchaksaraei et al. 2015), and so on are examples of MCDM methods which are applied in different situations. Sort of methods have been invented, developed and expanded conjoining with fuzzy theories or in any other scale of integration as hybrid MCDM models. The method Evaluation Based on Distance from Average Solution (EDAS) has been developed by Keshavarz Ghorabaee et al. (2015). TOPSIS and VIKOR are among top and most popular MCDM methods (Behzadian et al. 2012; Yazdani, Payam 2015). The logical function of these methods considers the optimal solution based on maximum distance from negative solution simultaneously minimum distance from best or positive ideal solution. However, the best alternative in the EDAS method is corresponded to the distance from average solution (AV). In this work we first present the crisp and ordinary fuzzy EDAS methods and then extend to its intuitionistic fuzzy version.

In EDAS method, first two measures are delivered as the positive distance from average (PDA), and the negative distance from average (NDA). These measures can show the difference between each solution (alternative) and the average solution. Therefore higher values of PDA and lower values of NDA will indicate optimal solution. In fact, the higher values of PDA and/or lower values of NDA represent that the solution (alternative) is better than average solution. Classical algorithm of EDAS can be followed using the following steps:

Step 1. Choose the most relevant attributes which describe decision alternatives for specific decision problem. 
Step 2. If $x_{i j}$ is the performance rating of $i^{\text {th }}$ alternative $A_{1}, A_{2}, \ldots, A_{n},(i=1,2, \ldots, n)$ respecting to the $j^{\text {th }}$ criterion $C_{1}, C_{2}, \ldots, C_{m}(j=1,2, \ldots, m)$. So, to form the interval decision matrix $X$ and weight of each criterion, following table and variables should be considered:

$$
\begin{gathered}
X=\left[x_{i j}\right]_{n \times m}=\left[\begin{array}{cccc}
x_{11} & x_{12} & \ldots & x_{1 m} \\
x_{21} & x_{22} & \ldots & x_{2 m} \\
\cdot & \cdot & \cdot & \cdot \\
\cdot & \cdot & \cdot & \cdot \\
\cdot & . & . & \cdot \\
x_{n 1} & x_{n 2} & \ldots & x_{n m}
\end{array}\right] . \\
W=\left[w_{1}, w_{2}, \ldots ., w_{m}\right]
\end{gathered}
$$

for $(i=1,2, \ldots, n)$ and $(j=1,2, \ldots, m)$, where $w_{j}$ is the weight of criterion $j^{\text {th }}$.

Step 3. According to the definition of EDAS method, the average solution with respect to all criteria must be determined as shown following formulas:

$$
A V_{j}=\frac{\sum_{i=1}^{n} x_{i j}}{n} \text {. }
$$

Step 4. The positive distance from average $(P D A)$ and the negative distance from average $(N D A)$ matrixes need to be calculated in this step according to lower and upper values of matrix as shown:

and

$$
P D A_{i j}=\frac{\max \left(0,\left(x_{i j}-A V_{j}\right)\right)}{A V_{j}},
$$

$$
N D A_{i j}=\frac{\max \left(0,\left(A V_{j}-x_{i j}\right)\right)}{A V_{j}} .
$$

In this way $P D A_{i j}$ and $N D A_{i j}$ represent the positive and negative distance of $i^{\text {th }}$ alternative from average solution in terms of $j^{\text {th }}$ criterion for the lower level of decision matrix, respectively.

Step 5. Obtain weighted summation of the positive distance and negative distances from average matric:

and

$$
S P_{i}=\sum_{j=1}^{m} w_{j} P D A_{i j}
$$

$$
S N_{i}=\sum_{j=1}^{m} w_{j} N D A_{i j} .
$$

Step 6. Identify the normalized values of $S P_{i}$ and $S N_{i}$ for all alternatives, shown as follows:

and

$$
N S P_{i}=\frac{S P_{i}}{\operatorname{Max}_{i}\left(S P_{i}\right)},
$$

$$
N S N_{i}=1-\frac{S N_{i}}{\operatorname{Max}_{i}\left(S N_{i}\right)} .
$$

Step 7. Detect the appraisal score $A S$ for all alternatives, shown as follows:

$$
A S_{i}=\frac{1}{2}\left(N S P_{i}+N S N_{i}\right),
$$

where $0 \leq A S_{i} \leq 1$.
Step 8. Rank the alternatives according to the decreasing values of appraisal score $\left(A S_{i}\right)$. The alternative with the highest $A S_{i}$ is the best choice among the candidate alternatives.

\section{Fuzzy extension of EDAS}

Fuzzy decision making models alongside development of MCDM methods are growing rapidly. The crucial importance of fuzzy decision models is evidence among research groups. Different MCDM methods with different instruments transformed to a fuzzy model. Fuzzy TOPSIS, fuzzy VIKOR, fuzzy COPRAS and etc. are examples in this area. In the following, ordinary fuzzy EDAS method developed by Keshavarz Ghorabaee et al. (2016) will be introduced. Later Intuitionistic fuzzy EDAS method is proposed. We first give the preliminaries for the ordinary fuzzy EDAS (Keshavarz Ghorabaee et al. 2016).

Definition 1. Let $\tilde{A}=\left(a_{1}, a_{2}, a_{3}, a_{4}\right)$ be a trapezoidal fuzzy number. The defuzzified value of $\tilde{A}$ can be obtained by Eq. 10:

$$
\kappa(\tilde{A})=\frac{1}{3}\left(a_{1}+a_{2}+a_{3}+a_{4}-\frac{a_{3} a_{4-} a_{1} a_{2}}{a_{3}+a_{4}-a_{1}-a_{2}}\right) .
$$

Definition 2. Suppose that $\tilde{A}=\left(a_{1}, a_{2}, a_{3}, a_{4}\right)$ is a trapezoidal fuzzy number. The function $\psi$ represents the maximum between $\tilde{A}$ and zero:

$$
\psi(\tilde{A})=\left\{\begin{array}{l}
\tilde{A}, \text { if } \kappa(\tilde{A})>0 \\
0, \text { if } \kappa(\tilde{A}) \leq 0
\end{array} .\right.
$$

\subsection{Ordinary fuzzy EDAS (Keshavarz Ghorabaee et al. 2016)}

Let us assume the fuzzy decision matrix as this:

$$
\tilde{X}=\left[\tilde{x}_{i j}\right]_{n \times m}=\left[\begin{array}{cccc}
\tilde{x}_{11} & \tilde{x}_{12} & \ldots & \tilde{x}_{1 m} \\
\tilde{x}_{21} & \tilde{x}_{22} & \ldots & \tilde{x}_{2 m} \\
\cdot & \cdot & . & \cdot \\
\cdot & \cdot & \cdot & \cdot \\
\cdot & \cdot & . & \cdot \\
\tilde{x}_{n 1} & \tilde{x}_{n 2} & \ldots & \tilde{x}_{n m}
\end{array}\right] .
$$

Consider $i^{\text {th }}$ alternative $A_{1}, A_{2}, \ldots, A_{n},(i=1,2, \ldots ., n)$ respecting to the $j^{\text {th }}$ criterion, so each member of decision matrix is denoted by triangular fuzzy number $\tilde{x}_{i j}=\left(x_{i j 1}, x_{i j 2}, x_{i j 3}\right)$. For each criterion weights are stated as $\tilde{w}=\left(w_{1}, w_{2}, w_{3}\right)$. So, to solve fuzzy EDAS model following steps must be pursued:

Step 1. Determine the fuzzy average decision matrix regarding to all of the criteria:

$$
\widetilde{A V}_{j}=\frac{\sum_{i=1}^{n} \tilde{x}_{i j}}{n} .
$$

Step 2. The optimal solution should have maximum distance from negative feasible solutions while as the same 
time minimum distance by best and ideal solutions, so, identify the fuzzy positive distance from average and the fuzzy negative distance from average:

$$
P D A=\left[\widetilde{p d a}_{i j}\right]_{n \times m},
$$

and

and

$$
N D A=\left[\widetilde{n d a_{i j}}\right]_{n \times m},
$$

$$
\widetilde{p d a_{i j}}=\left\{\begin{array}{l}
\frac{\psi\left(\tilde{x}_{i j} \ominus \widetilde{a v}_{j}\right)}{\kappa\left(\widetilde{a v} \tilde{v}_{j}\right)}, \text { if } j \in B \\
\frac{\psi\left(\widetilde{a v}_{j}-\tilde{x}_{i j}\right)}{\kappa\left(\widetilde{a v}_{j}\right)}, \text { if } j \in C
\end{array},\right.
$$

and

$$
\widetilde{n d a_{i j}}=\left\{\begin{array}{l}
\frac{\psi\left(\widetilde{a v}{ }_{j}-\tilde{x}_{i j}\right)}{\kappa\left(\widetilde{a v}{ }_{j}\right)}, \text { if } j \in B \\
\frac{\psi\left(\tilde{x}_{i j} \ominus \widetilde{a v}_{j}\right)}{\kappa\left(\widetilde{a v}{ }_{j}\right)}, \text { if } j \in C
\end{array} .\right.
$$

Step 3. Compute the fuzzy weighted summation of the positive distance and negative distances from average matric:

$$
\widetilde{s p}_{i}=\oplus_{j=1}^{m}\left(\tilde{w}_{j} \oplus \widetilde{p d a_{i j}}\right)
$$

and

$$
\widetilde{s n_{i}}=\oplus_{j=1}^{m}\left(\tilde{w}_{j} \oplus \widetilde{n d a_{i j}}\right)
$$

Step 4. Determine the fuzzy normalized values of $\widetilde{S N}_{i}$ and $\widetilde{S P}_{i}$.

and

$$
\widetilde{n s p_{i}}=\frac{\widetilde{s p}_{i}}{\max _{i}\left(\kappa\left(\widetilde{s p_{i}}\right)\right)},
$$

$$
\widetilde{n s n_{i}}=1-\frac{\widetilde{s n_{i}}}{\max _{i}\left(\kappa\left(\widetilde{s n_{i}}\right)\right)} .
$$

Step 5. Calculate the fuzzy appraisal score for all alternatives. Then this score must be deffuzified and finally highest appraisal score is the best choice among the candidate alternatives:

$$
\widetilde{a s}{ }_{i}=\frac{\widetilde{n s p_{i}} \oplus \widetilde{n s n_{i}}}{2} .
$$

Step 6. Rank the alternatives according to the decreasing values of defuzzified appraisal scores. In other words, the alternative with the highest appraisal score is the best choice among the candidate alternatives.

\subsection{The proposed IVIF EDAS}

In the fuzzy set theory, the membership of an element to a fuzzy set is a single value between zero and one. However, the degree of non-membership of an element " $v_{\tilde{A}}(x)$ " in a fuzzy set may not be equal to " $1-\mu_{\tilde{A}}(x)$ " since there may be some hesitation degree. Therefore, a generalization of fuzzy sets was proposed by Atanassov (1986) as intuitionistic fuzzy sets (IFS) which incorporate the degree of hesitation, which is defined as " $1-\mu_{\tilde{A}}(x)-v_{\tilde{A}}(x)$ ".

Let $X \neq \varnothing$ be a given set. An intuitionistic fuzzy set in $X$ is an object $A$ given by

$$
\tilde{A}=\left\{x, \mu_{\tilde{A}}(x), v_{\tilde{A}}(x) ; x \in X\right\},
$$

where $\mu_{\tilde{A}}: X \rightarrow[0,1]$ and $v_{\tilde{A}}: X \rightarrow[0,1]$ satisfy the condition:

$$
0 \leq \mu_{\tilde{A}}(x)+v_{\tilde{A}}(x) \leq 1,
$$

for every $x \in X$. Hesitancy is equal to " $1-\left(\mu_{\tilde{A}}(x)+v_{\tilde{A}}(x)\right)$ ".

The definition of interval-valued intuitionistic fuzzy sets (IVIFS) is given as follows. Let $D \subseteq[0,1]$ be the set of all closed subintervals of the interval and $X$ be a universe of discourse. An interval-valued intuitionistic fuzzy set in $\tilde{A}$ over $\mathrm{X}$ is an object having the form:

$$
\tilde{A}=\left\{<x, \tilde{\mu}_{A}(x), \tilde{v}_{A}(x)>\mid x \in X\right\},
$$

where $\tilde{\mu}_{\tilde{A}} \rightarrow D \subseteq[0,1], \quad \tilde{v}_{\tilde{A}}(x) \rightarrow D \subseteq[0,1]$ with the condition $0 \leq \sup \tilde{\mu}_{\tilde{A}}(x)+\sup \tilde{v}_{\tilde{A}}(x) \leq 1, \quad \forall x \in X$.

The intervals $\tilde{\mu}_{\tilde{A}}(x)$ and $\tilde{v}_{\tilde{A}}(x)$ denote the membership function and the non-membership function of the element $\mathrm{x}$ to the set $\mathrm{A}$, respectively. Thus, for each $x \in X$, $\tilde{\mu}_{\tilde{A}}(x)$ and $\tilde{v}_{\tilde{A}}(x)$ are closed intervals and their lower and upper end points are denoted by $\tilde{\mu}_{\widetilde{A L}}(x), \tilde{\mu}_{\widetilde{A U}}(x)$, $\tilde{v}_{\widetilde{A L}}(x)$, and $\tilde{v}_{\widetilde{A U}}(x)$, respectively. Interval-valued intiutionistic fuzzy set $\tilde{A}$ is then denoted by

$$
\tilde{A}=\left\{<x,\left[\tilde{\mu}_{\widetilde{A L}}(x), \tilde{\mu}_{\widetilde{A U}}(x)\right],\left[\tilde{v}_{\widetilde{A L}}(x), \tilde{v}_{\widetilde{A U}}(x)\right]>\mid x \in X\right\},
$$

where

$$
0 \leq \tilde{\mu}_{\widetilde{A U}}(x)+\tilde{v}_{\widetilde{A U}}(x) \leq 1, \tilde{\mu}_{\widetilde{A L}}(x) \geq 0, \tilde{v}_{\widetilde{A L}}(x) \geq 0 .
$$

For each element $x$, we can compute the unknown degree (hesitancy degree) of an interval-valued intuitionistic fuzzy interval of $x \in X$ in $\tilde{A}$ defined as follows:

$$
\begin{aligned}
& \pi_{\tilde{A}(x)}=1-\tilde{\mu}_{\tilde{A}}(x)-\tilde{v}_{\tilde{A}}(x)= \\
& \left(\left[1-\tilde{\mu}_{\widetilde{A U}}(x)-\tilde{v}_{\widetilde{A U}}(x)\right],\left[1-\tilde{\mu}_{\widetilde{A L}}(x)-\tilde{v}_{\widetilde{A L}}(x)\right]\right) .
\end{aligned}
$$

For convenience, let

$$
\tilde{\mu}_{\tilde{A}}(x)=\left[\mu^{-}, \mu^{+}\right], \tilde{v}_{\tilde{A}}(x)=\left[v^{-}, v^{+}\right],
$$

so $\tilde{A}=\left(\left[\mu^{-}, \mu^{+}\right],\left[v^{-}, v^{+}\right]\right)$. Figure 2 illustrates an interval-valued intuitionistic fuzzy set.

Some arithmetic operations with interval-valued intuitionistic fuzzy sets and $\lambda \geq 0$ are given in the following: Let $\tilde{I}_{1}=\left(\left[\mu_{1}^{-}, \mu_{1}^{+}\right],\left[v_{1}^{-}, v_{1}^{+}\right]\right)$and $\tilde{I}_{2}=\left(\left[\mu_{2}{ }^{-}, \mu_{2}{ }^{+}\right],\left[v_{2}{ }^{-}, v_{2}{ }^{+}\right]\right)$be two interval-valued intuitionistic fuzzy sets. Then,

$$
\tilde{I}_{1} \oplus \tilde{I}_{2}=\left(\left[\mu_{1}^{-}+\mu_{2}^{-}-\mu_{1}^{-} \mu_{2}^{-}, \mu_{1}^{+}+\mu_{2}^{+}-\mu_{1}^{+} \mu_{2}^{+}\right],\left[v_{1}^{-} v_{2}^{-}, v_{1}^{+} v_{2}^{+}\right]\right) ;
$$




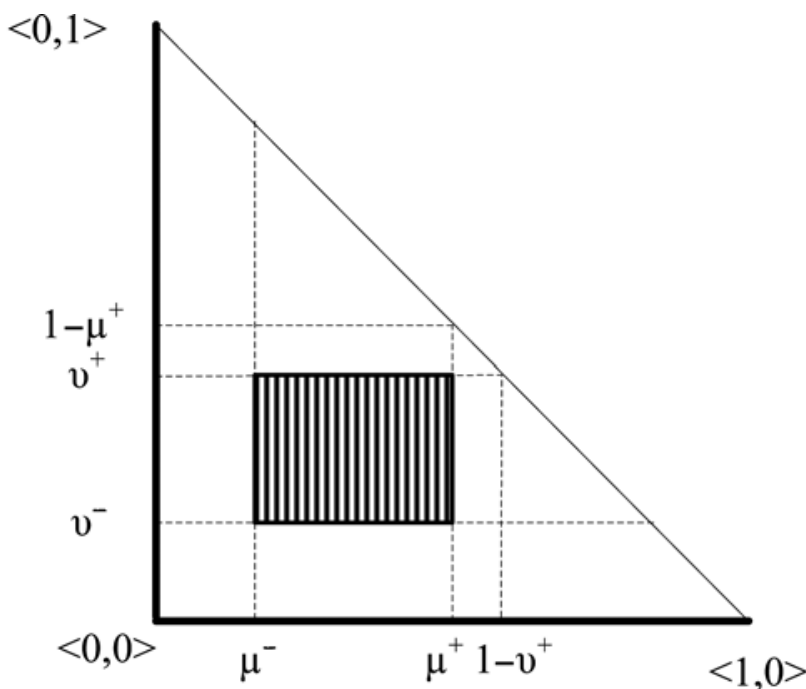

Fig. 2. Interval-valued intuitionistic fuzzy set

$$
\begin{gathered}
\tilde{I}_{1} \otimes \tilde{I}_{2}=\left(\left[\mu_{1}^{-} \mu_{2}^{-}, \mu_{1}^{+} \mu_{2}^{+}\right],\left[v_{1}^{-}+v_{2}^{-}-v_{1}^{-} v_{2}^{-}, v_{1}^{+}+v_{2}^{+}-v_{1}^{+} v_{2}^{+}\right]\right) ; \\
\tilde{I}_{1} \ominus \tilde{I}_{2}=\left\{\begin{array}{c}
\left(\left[\frac{\mu_{1}^{-}-\mu_{2}^{-}}{1-\mu_{2}^{-}}, \frac{\mu_{1}^{+}-\mu_{2}^{+}}{1-\mu_{2}^{+}}\right],\left[\frac{v_{1}^{-}}{v_{2}^{-}}, \frac{v_{1}^{+}}{v_{2}^{+}}\right]\right) \\
([0,0],[1,1]), \text { otherwise }
\end{array}\right.
\end{gathered}
$$

Eq. (28) is valid if $\mu_{1}^{-} \geq \mu_{2}^{-}, \mu_{1}^{+} \geq \mu_{2}^{+}, v_{1}^{-} \leq v_{2}^{-}, v_{1}^{+} \leq v_{2}^{+}$ and $\quad v_{2}^{-}>0, v_{2}^{+}>0$ and $v_{1}^{-}\left(1-\mu_{2}^{-}\right) \leq v_{2}^{-}\left(1-\mu_{1}^{-}\right)$, $v_{1}^{+}\left(1-\mu_{2}^{+}\right) \leq v_{2}^{+}\left(1-\mu_{1}^{+}\right)$.

$$
\tilde{I}_{1} \oslash \tilde{I}_{2}=\left\{\begin{array}{c}
\left(\left[\frac{\mu_{1}^{-}}{\mu_{2}^{-}}, \frac{\mu_{1}^{+}}{\mu_{2}^{+}}\right],\left[\frac{v_{1}^{-}-v_{2}^{-}}{1-v_{2}^{-}}, \frac{v_{1}^{+}-v_{2}^{+}}{1-v_{2}^{+}}\right]\right) \\
([0,0],[1,1]), \text { otherwise }
\end{array}\right.
$$

Eq. (29) is valid if $\mu_{1}^{-} \leq \mu_{2}^{-}, \mu_{1}^{+} \leq \mu_{2}^{+}, v_{1}^{-} \geq v_{2}^{-}, v_{1}^{+} \geq v_{2}^{+}$ and $\mu_{2}^{-}>0, \mu_{2}^{+}>0$ and $\mu_{1}^{-}\left(1-v_{2}^{-}\right) \leq \mu_{2}^{-}\left(1-v_{1}^{-}\right)$, $\mu_{1}^{+}\left(1-v_{2}^{+}\right) \leq \mu_{2}^{+}\left(1-v_{1}^{+}\right)$.

Multiplication by a fixed value, $\lambda \geq 0$, is given by Eq. (32).

$$
\lambda \tilde{I}_{1}=\left(\left[1-\left(1-\mu_{1}^{-}\right)^{\lambda}, 1-\left(1-\mu_{1}^{+}\right)^{\lambda}\right],\left[\left(v_{1}^{-}\right)^{\lambda},\left(v_{1}^{+}\right)^{\lambda}\right]\right) \text {. }
$$

The necessary definitions used in the proposed methodology are stated as follows:

Definition 3. Let $\tilde{\alpha}_{j}=\left(\left[\mu_{j}^{-}, \mu_{j}^{+}\right],\left[v_{j}^{-}, v_{j}^{+}\right]\right)$ $(j=1,2, \ldots, n)$ be a collection of interval-valued intuitionistic fuzzy numbers and let IIFWA: $Q^{n} \rightarrow Q$, if

$$
\operatorname{IIFWA}\left(\tilde{\alpha}_{1}, \tilde{\alpha}_{2}, \ldots, \tilde{\alpha}_{n}\right)=w_{1} \tilde{\alpha}_{1} \oplus w_{2} \tilde{\alpha}_{2} \oplus \ldots \oplus w_{n} \tilde{\alpha}_{n} .
$$

Then, IIFWA is called an interval-valued intuitionistic fuzzy weighted averaging (IIFWA) operator, where
$Q$ is the set of all IVIFNs, $w=\left(w_{1}, w_{2}, \ldots, w_{n}\right)$ is the weight vector of the IVIFNs $\tilde{\alpha}_{j}(j=1,2, \ldots, n)$, and $w_{j}>0$, $\sum_{j=1}^{n} w_{j}=1$. The IIFWA operator can be further transformed in to the following form:

$$
\begin{aligned}
& \operatorname{IIFWA}\left(\tilde{\alpha}_{1}, \tilde{\alpha}_{2}, \ldots, \tilde{\alpha}_{n}\right)=\left(\left[1-\prod_{j=1}^{n}\left(1-\mu_{j}^{-}\right)^{w_{i}},\right.\right. \\
& \left.\left.1-\prod_{j=1}^{n}\left(1-\mu_{j}^{+}\right)^{w_{i}}\right],\left[\prod_{j=1}^{n}\left(v_{j}^{-}\right)^{w_{i}}, \prod_{j=1}^{n}\left(v_{j}^{+}\right)^{w_{i}}\right]\right)
\end{aligned}
$$

Especially if $w=(1 / n, 1 / n, \ldots, 1 / n)$, then the IIFWA operator reduces to an interval-valued intuitionistic fuzzy averaging (IIFA) operator, where

$$
\begin{aligned}
& \operatorname{IIFA}\left(\tilde{\alpha}_{1}, \tilde{\alpha}_{2}, \ldots, \tilde{\alpha}_{n}\right)=\frac{1}{n}\left(\tilde{\alpha}_{1} \oplus \tilde{\alpha}_{2} \oplus \ldots \oplus \tilde{\alpha}_{n}\right)= \\
& \left(\left[1-\prod_{j=1}^{n}\left(1-\mu_{j}^{-}\right)^{1 / n}, 1-\prod_{j=1}^{n}\left(1-\mu_{j}^{+}\right)^{1 / n}\right],\left[\prod_{j=1}^{n}\left(v_{j}^{-}\right)^{1 / n}, \prod_{j=1}^{n}\left(v_{j}^{+}\right)^{1 / n}\right]\right) .
\end{aligned}
$$

In the following, the steps of the proposed intervalvalued intuitionistic fuzzy EDAS method are given:

Step 1. Choose the most relevant attributes which describe decision alternatives for specific decision problem.

Step 2. If $x_{i j}$ is the performance rating of $i^{\text {th }}$ alternative $A_{1}, A_{2}, \ldots, A_{n},(i=1,2, \ldots, n)$ respecting to the $j^{\text {th }}$ criterion $C_{1}, C_{2}, \ldots, C_{m}(j=1,2, \ldots, m)$, So, to form the interval decision matrix $X$ and weight of each criterion, following table and variables should be considered;

$$
\begin{aligned}
& X=\left[x_{i j}\right]_{n \times m}=\left[\begin{array}{cccc}
\tilde{x}_{11} & \tilde{x}_{12} & \ldots & \tilde{x}_{1 m} \\
\tilde{x}_{21} & \tilde{x}_{22} & \ldots & \tilde{x}_{2 m} \\
\cdot & \cdot & \cdot & \cdot \\
\cdot & \cdot & \cdot & \cdot \\
\tilde{x}_{n 1} & \tilde{x}_{n 2} & . & \tilde{x}_{n m}
\end{array}\right]= \\
& {\left[\begin{array}{c}
\left(\left[\mu_{11}^{-}, \mu_{11}^{+}\right],\left[v_{11}^{-}, v_{11}^{+}\right]\right)\left(\left[\mu_{12}^{-}, \mu_{12}^{+}\right],\left[v_{12}^{-}, v_{12}^{+}\right]\right) \ldots \\
\left(\left[\mu_{21}^{-}, \mu_{21}^{+}\right],\left[v_{21}^{-}, v_{21}^{+}\right]\right)\left(\left[\mu_{22}^{-}, \mu_{22}^{+}\right],\left[v_{22}^{-}, v_{22}^{+}\right]\right) \ldots \\
\cdot \\
\cdot \\
\cdot \\
\left(\left[\mu_{n 1}^{-}, \mu_{n 1}^{+}\right],\left[v_{n 1}^{-}, v_{n 1}^{+}\right]\right)\left(\left[\mu_{n 2}^{-}, \mu_{n 2}^{+}\right],\left[v_{n 2}^{-}, v_{n 2}^{+}\right]\right) \ldots \\
\left(\left[\mu_{1 m}^{-}, \mu_{1 m}^{+}\right],\left[v_{1 m}^{-}, v_{1 m}^{+}\right]\right) \\
\left(\left[\mu_{2 m}^{-}, \mu_{2 m}^{+}\right],\left[v_{2 m}^{-}, v_{2 m}^{+}\right]\right) \\
\cdot \\
\cdot \\
\left(\left[\mu_{n m}^{-}, \mu_{n m}^{+}\right],\left[v_{n m}^{-}, v_{n m}^{+}\right]\right)
\end{array}\right]}
\end{aligned}
$$


where

$$
\begin{aligned}
& \tilde{W}=\left(\tilde{w}_{1}, \tilde{w}_{2}, \ldots ., \tilde{w}_{m}\right)=\left(\left(\left[\mu_{w 1}^{-}, \mu_{w 1}^{+}\right],\left[v_{w 1}^{-}, v_{w 1}^{+}\right]\right),\right. \\
& \left.\left(\left[\mu_{w 2}^{-}, \mu_{w 2}^{+}\right],\left[v_{w 2}^{-}, v_{w 2}^{+}\right]\right), \ldots,\left(\left[\mu_{w m}^{-}, \mu_{w m}^{+}\right],\left[v_{w m}^{-}, v_{w m}^{+}\right]\right)\right)
\end{aligned}
$$

and $\tilde{w}_{j}$ is the weight of criterion $j ;(i=1,2, \ldots, n)$ and $(j=1,2, \ldots, m)$.

Step 3. According to the definition of EDAS method, the average solution with respect to all criteria must be determined as shown in Eq. (37):

$\tilde{A V_{j}}=\frac{\left(\left[\sum_{i=1}^{n} \mu_{i j}^{-}-\sum_{\substack{i=1 \\ i \neq k}}^{n} \mu_{i j}^{-} \times \mu_{k j}^{-}, \sum_{i=1}^{n} \mu_{i j}^{+}-\sum_{\substack{i=1 \\ i \neq k}}^{n} \mu_{i j}^{+} \times \mu_{k j}^{+}\right],\left[\prod_{i=1}^{n} v_{i j}^{-}, \prod_{i=1}^{n} v_{i j}^{+}\right]\right)}{n}$, $j=1,2, \ldots, m$,

Or

$\tilde{A V_{j}}=\left(\left[\left(1-\sum_{i=1}^{n} \mu_{i j}^{-}+\sum_{\substack{i=1 \\ i \neq k}}^{n} \mu_{i j}^{-} \times \mu_{k j}^{-}\right)^{1 / n}\right.\right.$,

$\left.\left.\left(1-\sum_{i=1}^{n} \mu_{i j}^{+}+\sum_{\substack{i=1 \\ i \neq k}}^{n} \mu_{i j}^{+} \times \mu_{k j}^{+}\right)^{1 / n}\right],\left[\left(\prod_{i=1}^{n} v_{i j}^{-}\right)^{1 / n},\left(\prod_{i=1}^{n} v_{i j}^{+}\right)^{1 / n}\right]\right)$.

Step 4. The positive distance from average $(P D A)$ and the negative distance from average (NDA) matrixes need to be calculated in this step according to lower and upper values of matrix as below:

$$
\underset{P D A_{i j}}{\sim} \frac{\max \left(0,\left(\left[\mu_{i j}^{-}, \mu_{i j}^{+}\right],\left[v_{i j}^{-}, v_{i j}^{+}\right]\right)-\tilde{A V} j\right)}{\kappa(\tilde{A V j})},
$$

and

$$
\tilde{N D A_{i j}}=\frac{\max \left(0, \tilde{A V_{j}}-\left(\left[\mu_{i j}^{-}, \mu_{i j}^{+}\right],\left[v_{i j}^{-}, v_{i j}^{+}\right]\right)\right)}{\kappa\left(\tilde{A V_{j}}\right)} .
$$

In this way $P D A_{i j}$ and $N D A_{i j}$ represent the positive and negative distance of $i^{\text {th }}$ alternative from average solution in terms of $j^{\text {th }}$ criterion for the lower level of decision matrix, respectively.

We propose the following ranking method for determining the maximum term in Eqs (39) and (40). Let $\tilde{\alpha}=([a, b],[c, d])$ be an interval-valued intutionistic fuzzy number. The following score function is proposed for defuzzifying $\tilde{\alpha}$ :

$$
I(\tilde{\alpha})=\frac{a+b+(1-c)+(1-d)+a \times b-\sqrt{(1-c) \times(1-d)}}{4} .
$$

In Eq. (41), the terms $(1-\mathrm{c})$ and $(1-\mathrm{d})$ convert nonmembership degrees to membership degrees while the term $\sqrt{(1-c) \times(1-d)}$ decreases the defuzzified value.

Step 5. Obtain weighted summation of the positive and negative distances from average matrix:

$$
\begin{aligned}
& \stackrel{\sim}{S P_{i}}=\left(\left[\sum_{j=1}^{m} \mu_{w_{j}}^{-} \times \mu_{P D A_{i j}}^{-}, \sum_{j=1}^{m} \mu_{w_{j}}^{+} \times \mu_{P D A_{i j}}^{+}\right],\right. \\
& \left.\left[\sum_{j=1}^{m} v_{w_{j}}^{-}-\sum_{\substack{j=1 \\
j \neq k}}^{m} v_{w_{j}}^{-} \times v_{P D A_{k j}}^{-}, \sum_{j=1}^{m} v_{w_{j}}^{+}-\sum_{\substack{j=1 \\
j \neq k}}^{m} v_{w_{j}}^{+} \times v_{P D A_{k j}}^{+}\right]\right), i=1,2, \ldots, n,
\end{aligned}
$$

and

$$
\begin{aligned}
& \stackrel{\sim}{S N_{i}}=\left(\left[\sum_{j=1}^{m} \mu_{w_{j}}^{-} \times \mu_{N D A_{i j}}^{-}, \sum_{j=1}^{m} \mu_{w_{j}}^{+} \times \mu_{N D A_{i j}}^{+}\right],\right. \\
& \left.\left[\sum_{j=1}^{m} v_{w_{j}}^{-}-\sum_{\substack{j=1 \\
j \neq k}}^{m} v_{w_{j}}^{-} \times v_{N D A_{k j}}^{-}, \sum_{j=1}^{m} v_{w_{j}}^{+}-\sum_{\substack{j=1 \\
j \neq k}}^{m} v_{w_{j}}^{+} \times v_{N D A_{k j}}^{+}\right]\right)
\end{aligned}
$$$$
i=1,2, \ldots, n \text {. }
$$

Step 6. Identify the normalized values of $\tilde{S P_{i}}$ and $S N_{i}$ for all alternatives. A defuzzification process is needed to select the maximum $S P_{i}$ and $S N_{i}$. The defuzzification equation given in Step 4 is reused for this aim. Based on the division operation between IVIFNs, intuitionistic $N S P_{i}^{\text {intu }}$ and $N S N_{i}^{\text {intu }}$ are obtained as follows:

$$
\begin{aligned}
& \tilde{N S P}{ }_{i}^{i n t u}= \\
& \left(\left[\frac{\sum_{j=1}^{m} \mu_{w_{j}}^{-} \times \mu_{P D A_{i j}}^{-}}{\operatorname{Max}\left(\sum_{j=1}^{m} \mu_{w_{j}}^{+} \times \mu_{P D A_{i j}}^{+}\right)}, \frac{\sum_{j=1}^{m} \mu_{w_{j}}^{+} \times \mu_{P D A_{i j}}^{+}}{\operatorname{Max}\left(\sum_{j=1}^{m} \mu_{w_{j}}^{+} \times \mu_{P D A_{i j}}^{+}\right)}\right)\right. \\
& \underline{\sum_{j=1}^{m} v_{w_{j}}^{-}-\sum_{\substack{j=1 \\
j \neq k}}^{m} v_{w_{j}}^{-} \times v_{P D A_{k j}}^{-}-\operatorname{Max}\left(\sum_{j=1}^{m} v_{w_{j}}^{-}-\sum_{\substack{j=1 \\
j \neq k}}^{m} v_{w_{j}}^{-} \times v_{P}^{\bar{P}} D A_{k j}\right)} \\
& 1-\operatorname{Max}\left(\sum_{j=1}^{m} v_{w_{j}}^{-}-\sum_{\substack{j=1 \\
j \neq k}}^{m} v_{w_{j}}^{-} \times v_{P D A_{k j}}^{-}\right)
\end{aligned}
$$

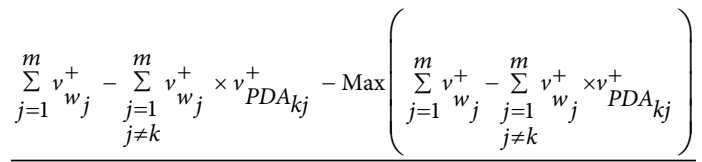

$$
\begin{aligned}
& 1-\operatorname{Max}\left(\sum_{j=1}^{m} v_{w_{j}}^{+}-\sum_{\substack{j=1 \\
j \neq k}}^{m} v_{w_{j}}^{+} \times v_{P D A_{k j}}^{+}\right)
\end{aligned}
$$


and

$$
\begin{aligned}
& \tilde{N S N}_{i}^{\text {intu }}=1- \\
& \left(\left[\frac{\sum_{j=1}^{m} \mu_{w_{j}}^{-} \times \mu_{N D A_{i j}}^{-}}{\operatorname{Max}\left(\sum_{j=1}^{m} \mu_{w_{j}}^{+} \times \mu_{N D A_{i j}}^{+}\right)}, \frac{\sum_{j=1}^{m} \mu_{w_{j}}^{+} \times \mu_{N D A_{i j}}^{+}}{\operatorname{Max}\left(\sum_{j=1}^{m} \mu_{w_{j}}^{+} \times \mu_{N D A_{i j}}^{+}\right)}\right],\right. \\
& \sum_{j=1}^{m} v_{w_{j}}^{-}-\sum_{\substack{j=1 \\
j \neq k}}^{m} v_{w_{j}}^{-} \times v_{N D A_{k j}}^{-}-\operatorname{Max}\left(\sum_{j=1}^{m} v_{w_{j}}^{-}-\sum_{\substack{j=1 \\
j \neq k}}^{m} v_{w_{j}}^{-} \times v_{N}^{-} A_{k j}\right) \\
& 1-\operatorname{Max}\left(\sum_{j=1}^{m} v_{w_{j}}^{-}-\sum_{\substack{j=1 \\
j \neq k}}^{m} v_{w_{j}}^{-} \times v_{N D A_{k j}}^{-}\right) \\
& \sum_{j=1}^{m} v_{w_{j}}^{+}-\sum_{\substack{j=1 \\
j \neq k}}^{m} v_{w_{j}}^{+} \times v_{N D A_{k j}}^{+}-\operatorname{Max}\left(\sum_{j=1}^{m} v_{w_{j}^{+}}^{+}-\sum_{\substack{j=1 \\
j \neq k}}^{m} v_{w_{j}}^{+} \times v_{N D A}^{+} A_{k j}\right) \\
& 1-\operatorname{Max}\left(\sum_{j=1}^{m} v_{w_{j}}^{m}-\sum_{\substack{j=1 \\
j \neq k}}^{m} v_{w_{j}^{+}}^{+} \times v_{N D A_{k j}}^{+}\right)
\end{aligned}
$$

Step 7. Detect the intuitionistic appraisal score $A S^{\text {intu }}$ for all alternatives, shown as follows:

$$
\tilde{A} \tilde{S^{i n t u}}=\frac{1}{2}\left(\tilde{N S P}{ }_{i}^{i n t u}+\tilde{N S} N_{i}^{i n t u}\right),
$$

where $0 \leq A S^{\text {intu }} \leq 1$.

Step 8. Rank the alternatives according to the decreasing values of appraisal score $\left(A S^{i n t u}\right)$. The alternative with the highest $A S^{i n t u}$ is the best choice among the candidate alternatives.

\section{Application}

Various environmental protection problems have been solved by using multi criteria decision making techniques (Turskis et al. 2012; Zavadskas et al. 2009; Khan, Samadder 2015). Solid waste disposal site selection is also an important environmental problem that we have to use many tangible, intangible, and conflicting criteria in its solution.

In a multicriteria solid waste disposal site selection problem, there are three alternatives and three criteria which are water pollution (W), distance to residential areas (D), and slope (S). The evaluation scales for crisp case, ordinary fuzzy case, and intuitionistic fuzzy case are given in Table 1.

\subsection{Application of Crisp EDAS}

The crisp decision matrix is given as in Table 2. All criteria are equally weighted.
Table 1. Evaluation scale

\begin{tabular}{cccc}
\hline $\begin{array}{c}\text { Linguis- } \\
\text { tic term }\end{array}$ & $\begin{array}{c}\text { Crisp } \\
\text { scale }\end{array}$ & $\begin{array}{c}\text { Ordinary fuzzy } \\
\text { scale }\end{array}$ & $\begin{array}{c}\text { Intuitionistic fuzzy } \\
\text { scale }\end{array}$ \\
\hline AH & 100 & $(0.80,0.90,1,1)$ & $([0.85,1.00],[0,0])$ \\
\hline VH & 85 & $(0.70,0.80,0.80,1)$ & $\begin{array}{c}([0.75,0.95], \\
[0,0.05])\end{array}$ \\
\hline \multirow{2}{*}{$\mathrm{H}$} & 70 & $\begin{array}{c}(0.50,0.60,0.70, \\
0.80)\end{array}$ & $\begin{array}{c}([0.60,0.80], \\
[0.05,0.20])\end{array}$ \\
\hline \multirow{2}{*}{$\mathrm{M}$} & \multirow{2}{*}{50} & $\begin{array}{c}(0.40,0.50,0.50, \\
0.60)\end{array}$ & $\begin{array}{c}([0.40,0.60], \\
[0.15,0.40])\end{array}$ \\
\hline \multirow{2}{*}{$\mathrm{L}$} & \multirow{2}{*}{30} & $\begin{array}{c}(0.20,0.30,0.40, \\
0.50)\end{array}$ & $\begin{array}{c}([0.05,0.20], \\
[0.60,0.80])\end{array}$ \\
\hline \multirow{2}{*}{$\mathrm{VL}$} & \multirow{2}{*}{20} & $\begin{array}{c}(0.10,0.20,0.20, \\
0.30)\end{array}$ & $\begin{array}{c}([0,0.05], \\
[0.75,0.95])\end{array}$ \\
\hline $\mathrm{AL}$ & 10 & $(0,0,0.10,0.20)$ & $([0,0],[0.85,1.00])$ \\
\hline
\end{tabular}

Table 2. Crisp Decision Matrix

\begin{tabular}{cccc}
\hline Alternatives & W & D & S \\
\hline SWDS-1 & 95 & 65 & 77 \\
\hline SWDS-2 & 80 & 85 & 74 \\
\hline SWDS-3 & 86 & 72 & 80 \\
\hline Average & 87 & 74 & 77 \\
\hline
\end{tabular}

Using Eqs (3) and (4), crisp PDA and NDA values are obtained as in Table 3.

Table 3. PDA and NDA values

\begin{tabular}{cccccc}
\hline $\begin{array}{c}\text { PDA } \\
j=1\end{array}$ & $\begin{array}{c}\text { PDA } \\
j=2\end{array}$ & $\begin{array}{c}\text { PDA } \\
j=3\end{array}$ & $\begin{array}{c}\text { NDA } \\
j=1\end{array}$ & $\begin{array}{c}\text { NDA } \\
j=2\end{array}$ & $\begin{array}{c}\text { NDA } \\
j=3\end{array}$ \\
\hline 0.092 & 0.000 & 0.000 & 0.000 & 0.000 & 0.000 \\
\hline 0.000 & 0.149 & 0.000 & 0.080 & 0.002 & 0.039 \\
\hline 0.000 & 0.000 & 0.039 & 0.011 & 0.000 & 0.000 \\
\hline
\end{tabular}

Using Eqs (5), (6), (7), and (8), $\mathrm{SP}_{\mathrm{i}}$ and $\mathrm{NSP}_{\mathrm{i}}$ values are obtained as in Table 4.

Table 4. $S P_{i}$ and $N S P_{i}$ values

\begin{tabular}{cccc}
\hline$S P_{i}$ & $S N_{i}$ & $N S P_{i}$ & $N S N_{i}$ \\
\hline 0.031 & 0.000 & 0.619 & 1.000 \\
\hline 0.050 & 0.040 & 0.999 & 0.000 \\
\hline 0.013 & 0.004 & 0.262 & 0.910 \\
\hline
\end{tabular}

Based on the values in Table 4, ranking of the alternatives are obtained as in Table 5.

Table 5. ASi values

\begin{tabular}{ccc}
\hline Alternative & $A S_{i}=\left(N S P_{i}+N S N_{i}\right) / 2$ & Rank \\
\hline SWDS-1 & 0.809 & 1 \\
\hline SWDS-2 & 0.499 & 3 \\
\hline SWDS-3 & 0.584 & 2 \\
\hline
\end{tabular}

SWDS-1 is the best of all. 


\subsection{Application of Ordinary Fuzzy EDAS}

In this case, the decision matrix in Table 6 is filled in by using the linguistic scale given in Table 1.

Table 6. Linguistic Decision Matrix

\begin{tabular}{cccc}
\hline Alternatives & W & D & S \\
\hline SWDS -1 & AH & H & VH \\
SWDS -2 & VH & VH & H \\
SWDS -3 & VH & H & VH \\
\hline
\end{tabular}

The fuzzy average for each criterion is calculated as follows:

$$
\begin{aligned}
& \widetilde{A V}{ }_{W}=(0.69,0.85,0.85,0.97) ; \\
& \widetilde{A V}_{D}=(0.61,0.79,0.79,0.91) ; \\
& \widetilde{A V}_{S}=(0.62,0.79,0.79,0.94) .
\end{aligned}
$$

The fuzzy positive and negative distances from average are found as follows:

\section{For SWDS-1,}

$\widehat{P D A}_{1 W}=(-0.20947,0.07031,0.21092,0.536983)$, $\widetilde{P D A}_{1 D}=(0, \quad 0,0,0), \widetilde{P D A}_{1 S}=(-0.32594, \quad 0.016159$, $0.016159,0.78243)$,

For SWDS-2,

$\widetilde{P D A}_{2 W}=(-0.33269,-0.07031,-0.07031,0.536983)$, $\widetilde{P D A}_{2 D}=(0,0,0,0), \widetilde{P D A}_{2 S}=(-0.32594,0.016159$, $0.016159,0.78243)$,

For SWDS-3,

$\widetilde{P D A}_{3 W}=(-0.33269,-0.07031,-0.07031,0.536983)$, $\widetilde{P D A}_{3 D}=(0,0,0,0), \widetilde{P D A}_{3 S}=(-0.32594,0.016159$, $0.016159,0.78243)$,

For SWDS-1,

$\widehat{N D A}_{1 W}=(0,0,0,0), \widehat{N D A}_{1 D}=(-0.24675,0.116883$, $0.246753,0.532468), \widehat{N D A}_{1 S}=(0,0,0,0)$,

For SWDS-2,

$\widehat{N D A}_{2 W}=(-0.37052,0.059761,0.059761,0.322709)$, $\widehat{P D A}_{2 D}=(-0.24675, \quad 0.116883,0.246753,0.532468)$, $\widetilde{P D A}_{2 S}=(0,0,0,0)$,

For SWDS-3,

$\widehat{N D A}_{3 W}=(-0.37052,0.059761,0.059761,0.322709)$, $\widetilde{P D A}_{3 D}=(-0.24675, \quad 0.116883,0.246753,0.532468)$, $\widetilde{P D A}_{3 S}=(0,0,0,0)$,

The fuzzy weighted summations of the positive distance and negative distances from average matrix using equal criteria weights are as follows:

$$
\begin{aligned}
& \widetilde{S P}_{1}=(-0.17669,0.028534,0.074937,0.435406) \\
& \widetilde{S N}_{1}=(-0.08143,0.038571,0.081429,0.175714) \\
& \widetilde{S P}_{2}=(-0.21735,-0.01787,-0.01787,0.435406) ; \\
& \widetilde{S N}_{2}=(-0.2037,0.058293,0.10115,0.282208)
\end{aligned}
$$

$$
\begin{gathered}
\widetilde{S P}_{3}=(-0.21735,-0.01787,-0.01787,0.435406) \\
\widetilde{S N}_{3}=(-0.2037,0.058293,0.10115,0.282208)
\end{gathered}
$$

The fuzzy normalized values of $\widetilde{S N}_{i}$ and $\widetilde{S P}_{i}$ are calculated as follows:

$$
\begin{aligned}
& \widetilde{N S P}_{1}=(-3.39836,0.548819,1.441324,8.374566) ; \\
& \widetilde{N S N}_{1}=(-2.37647,-0.56471,0.258824,2.564706) ; \\
& \widetilde{N S P}_{2}=(-4.18045,-0.34369,-0.34369,8.374566) ; \\
& \widetilde{N S N}_{2}=(-4.42283,-0.94366,-0.12013,4.914225) ; \\
& \widetilde{N S P}_{3}=(-4.18045,-0.34369,-0.34369,8.374566) ; \\
& \widetilde{N S N}_{3}=(-4.42283,-0.94366,-0.12013,4.914225) .
\end{aligned}
$$

The defuzzified appraisal score (as) for each alternative is calculated as follows:

$$
\begin{aligned}
& \operatorname{defuzz}\left(\widetilde{a s_{1}}\right)=0.974093 \\
& \operatorname{defuzz}\left(\widetilde{a s_{2}}\right)=0.615543 ; \\
& \operatorname{defuzz}\left(\widetilde{a s_{3}}\right)=0.615543 .
\end{aligned}
$$

The ranking of alternatives is as follows: $1>2=3$. The best alternative is the same as when the crisp EDAS was used. However, the other alternatives are equivalent when ordinary fuzzy EDAS is used. This is caused because of the vagueness in the linguistic evaluations.

\subsection{Application of Interval-Valued Intuitionistic Fuzzy EDAS}

In this case, the decision matrix is filled in by using the linguistic scale given in Table 1 as in Table 7.

Table 7. Intuitionistic Decision Matrix

\begin{tabular}{cccc}
\hline $\begin{array}{c}\text { Alter- } \\
\text { natives }\end{array}$ & $W$ & $D$ & $S$ \\
\hline \multirow{2}{*}{ SWDS-1 } & $([0.85,1.00]$, & $([0.60,0.80]$, & $([0.75,0.95]$, \\
& $[0,0])$ & $[0.05,0.20])$ & $[0,0.05])$ \\
\hline \multirow{2}{*}{ SWDS-2 } & $([0.75,0.95]$, & $([0.75,0.95]$, & $([0.60,0.80]$, \\
& $[0,0.05])$ & $[0,0.05])$ & $[0.05,0.20])$ \\
\hline \multirow{2}{*}{ SWDS-3 } & $([0.75,0.95]$, & $([0.60,0.80]$, & $([0.75,0.95]$, \\
& $[0,0.05])$ & $[0.05,0.20])$ & $[0,0.05])$ \\
\hline
\end{tabular}

The average values with respect to all criteria are determined as in Table 8:

Table 8. Average values with respect to criteria

\begin{tabular}{lc}
\hline $\mathrm{W}$ & $([0.78703,0.966383],[0,0])$ \\
\hline $\mathrm{D}$ & $([0.67679,0.848093],[0,0.125992])$ \\
\hline $\mathrm{S}$ & $([0.713022,0.897317],[0,0.07937])$ \\
\hline
\end{tabular}

PDA and NDA values of Alternatives 1, 2, and 3 are calculated as in Table 9. 
Table 9. PDA and NDA values of alternatives

\begin{tabular}{cc}
\hline$\widetilde{P D A}_{1 W}=([-0.13248,0.242425],[0,0])$ & $\widetilde{N D A}_{1 W}=([-0.24243,0.132479],[0,0])$ \\
\hline$\widetilde{P D A}_{1 D}=([-0.32665,0.162226],[-0.10006,0.263332])$ & $\widetilde{N D A}_{1 D}=([-0.16223,0.326654],[-0.26333,0.100056])$ \\
\hline$\widetilde{P D A}_{1 S}=([-0.1835,0.295182],[-0.09886,0.06228])$ & $\widetilde{N D A}_{1 S}=([-0.29518,0.1835],[-0.06228,0.098864])$ \\
\hline$\widetilde{P D A}_{2 W}=([-0.24631,0.18551],[0,0.056915])$ & $\widetilde{N D A}_{2 W}=([-0.18551,0.24631],[-0.05692,0])$ \\
\hline$\widetilde{P D A}_{2 D}=([-0.12915,0.359725],[-0.16589,0.065833])$ & $\widetilde{N D A}_{2 D}=([-0.35973,0.129155],[-0.06583,0.165889])$ \\
\hline$\widetilde{P D A}_{2 S}=([-0.37034,0.108341],[-0.03658,0.249122])$ & $\widetilde{N D A}_{2 S}=([-0.10834,0.370341],[-0.24912,0.036584])$ \\
\hline$\widetilde{P D A}_{3 W}=([-0.24631,0.18551],[0,0.056915])$ & $\widetilde{N D A}_{3 W}=([-0.18551,0.24631],[-0.05692,0])$ \\
\hline$\widetilde{P D A}_{3 D}=([-0.32665,0.162226],[-0.10006,0.263332])$ & $\widetilde{N D A}_{3 S}=([-0.29518,0.1835],[-0.06228,0.098864])$ \\
\hline
\end{tabular}

SP, NSP, and the defuzzified $\widetilde{a s}$ values are given below.

$$
\begin{aligned}
& \widetilde{S P}_{1}=([-0.21421,0.233278],[-0.06631,0.108538]) ; \\
& \widetilde{S N}_{1}=([-0.23328,0.214211],[-0.10854,0.066307]) \text {; } \\
& \widetilde{N S P}_{1}=([-0.82574,0.899241],[-0.2556,0.418392]) \text {; } \\
& \widehat{N S N}_{1}=([-0.86566,0.794904],[-0.40277,0.246054]) \text {; } \\
& \operatorname{defuzz}\left(\widetilde{a s_{1}}\right)=0.493021 \\
& \widetilde{S P}_{2}=([-0.2486,0.217859],[-0.06749,0.123957]) \text {; } \\
& \widetilde{S N}_{2}=([-0.21786,0.248602],[-0.12396,0.067491]) \text {; } \\
& \widetilde{N S P}_{2}=([-0.91305,0.800139],[-0.24788,0.455261]) \text {; } \\
& \widehat{N S N}_{2}=([-0.77849,0.888345],[-0.44294,0.241169]) \text {; } \\
& \operatorname{defuzz}\left(\widetilde{a s}_{2}\right)=0.443874 \text {; } \\
& \widetilde{S P}_{3}=([-0.25215,0.214306],[-0.06631,0.127509]) ; \\
& \widetilde{S N}_{3}=([-0.21431,0.252155],[-0.12751,0.066307]) ; \\
& \widetilde{N S P}_{3}=([-0.97201,0.826109],[-0.2556,0.491524]) \text {; } \\
& \widehat{N S N}_{3}=([-0.79526,0.935707],[-0.47317,0.246054]) \\
& \operatorname{defuzz}\left(\widetilde{a s_{3}}\right)=0.430828 \text {. }
\end{aligned}
$$

According to the obtained results, the ranking of the alternatives is $1>2>3$. The best alternative is again SWDS-1. However, the second best alternative is SWDS-2 with intuitionistic fuzzy EDAS where as it is 3 with crisp
EDAS. Alternatives SWDS-2 and SWDS-3 are equivalent with ordinary fuzzy EDAS. These differences come from the structures of the data, which are exact in crisp case, trapezoidal in ordinary fuzzy case, and interval-valued in intuitionistic fuzzy case. A decision maker should select the proper approach with respect to the data he/she has.

\subsection{Sensitivity analysis}

The weights of the alternatives have been significantly changed as it can be seen in Table 10. However, the ranking has not changed. SWDS-1 is the best alternative in all cases. Alternative 2 is the second best alternative in all cases.

Table 10 . Sensitivity analysis by changing criteria weights

\begin{tabular}{cc}
\hline Weights & Rankings of alternatives \\
\hline$w_{w}=0.3, w_{D}=0.35, w_{S}=0.35$ & $\begin{array}{c}1>2>3\left(A S_{1}=0.495,\right. \\
\left.A S_{2}=0.449, A S_{3}=0.438\right)\end{array}$ \\
\hline$w_{w}=0.4, w_{D}=0.4, w_{S}=0.2$ & $\begin{array}{c}1>2>3\left(A S_{1}=0.489,\right. \\
\left.A S_{2}=0.433, A S_{3}=0.416\right)\end{array}$ \\
\hline$w_{w}=0.1, w_{D}=0.3, w_{S}=0.6$ & $\begin{array}{c}1>2>3\left(A S_{1}=0.499,\right. \\
\left.A S_{2}=0.483, A S_{3}=0.480\right)\end{array}$ \\
\hline$w_{w}=0.6, w_{D}=0.2, w_{S}=0.2$ & $\begin{array}{c}1>2>3\left(A S_{1}=0.472,\right. \\
\left.S_{2}=0.400, A S_{3}=0.371\right)\end{array}$ \\
\hline$w_{w}=0.1, w_{D}=0.8, w_{S}=0.1$ & $\begin{array}{c}1>2>3\left(A S_{1}=0.499,\right. \\
\left.A S_{2}=0.483, A S_{3}=0.480\right)\end{array}$ \\
\hline$w_{w}=0.4, w_{D}=0.2, w_{S}=0.4$ & $\begin{array}{c}1>2>3\left(A S_{1}=0.489,\right. \\
\left.A S_{2}=0.433, A S_{3}=0.416\right)\end{array}$ \\
\hline
\end{tabular}

Table 10 indicates that the ranking result obtained by IVIF EDAS is very robust. From slight changes to extreme changes in criteria weights, SWDS-1 is selected without any doubt. Smaller weights of the criterion "water pollution $(W)$ " cause the scores of alternatives 2 and 3 get closer. 
We also checked the effect of a change in linguistic evaluations to the final decision. For this aim, the linguistic evaluations of SWDS-1 have been changed from $\mathrm{AH}$, $\mathrm{H}, \mathrm{VH}$ to $\mathrm{AH}, \mathrm{H}, \mathrm{H}$. This change produces the following defuzzified appraisal scores (as): $\operatorname{defuzz}\left(\widetilde{a s}_{1}\right)=0.356$, $\operatorname{defuzz}\left(\widetilde{a s}_{1}\right)=0.444$, and $\operatorname{defuzz}\left(\widetilde{a s}_{1}\right)=0.432$. SWDS-2 becomes the best alternative in this case.

\section{Conclusions}

EDAS method needs fewer computations with respect to most of the other multiattribute decision-making methods while it can produce the same ranking of alternatives. The evaluation of alternatives in this method is based on distances of each alternative from the average solution with respect to each criterion. Ordinary fuzzy extension of crisp EDAS has been developed based on trapezoidal fuzzy numbers. We have proposed the interval-valued intuitionistic fuzzy EDAS (IVIF EDAS) method in this paper. The linguistic evaluations have been represented by interval-valued intuitionistic fuzzy sets and their arithmetic operations have been applied. Since the vagueness included by trapezoidal fuzzy sets and interval-valued intuitionistic fuzzy sets are basically different, the obtained rankings may change from ordinary fuzzy EDAS (OF EDAS) to intuitionistic fuzzy EDAS. The calculations of IVIF EDAS are more tedious with respect to ordinary fuzzy EDAS. Different ranking equations must be used in OF EDAS and IVIF EDAS.

For further research, the other extensions of fuzzy sets such as type-2 fuzzy sets, hesitant fuzzy sets, or neutrosophic sets may be used to develop the other versions of fuzzy EDAS.

\section{References}

Atanassov, K. T. 1986. Intuitionistic fuzzy sets, Fuzzy Sets and Systems 20: 87-96. https://doi.org/10.1016/S0165-0114(86)80034-3

Atanassov, K. T.; Gargov, G. 1989. Interval valued intuitionistic fuzzy sets, Fuzzy sets and systems 31(3): 343-349. https://doi.org/10.1016/0165-0114(89)90205-4

Behzadian, M.; Otaghsara, S. K.; Yazdani, M.; Ignatius, J. 2012. A state-of the-art survey of TOPSIS applications, Expert Systems with Applications 39(17): 13051-13069.

https://doi.org/10.1016/j.eswa.2012.05.056

Büyüközkan, G.; Çifçi, G. 2012. A novel hybrid MCDM approach based on fuzzy DEMATEL, fuzzy ANP and fuzzy TOPSIS to evaluate green suppliers, Expert Systems with Applications 39(3): 3000-3011.

https://doi.org/10.1016/j.eswa.2011.08.162

Cevik Onar, S.; Oztaysi, B.; Otay, İ; Kahraman, C. 2015. Multiexpert wind energy technology selection using interval-valued intuitionistic fuzzy sets, Energy 90: 274-285. https://doi.org/10.1016/j.energy.2015.06.086
Chakraborty, S.; Zavadskas, E. K. 2014. Applications of WASPAS method in manufacturing decision making, Informatica 25(1): 1-20. https://doi.org/10.15388/Informatica.2014.01

Corrente, S.; Greco, S.; Słowiński, R. 2013. Multiple criteria hierarchy process with ELECTRE and PROMETHEE, Omega 41(5): $820-846$.

https://doi.org/10.1016/j.omega.2012.10.009

Deng, X.; Hu, Y.; Deng, Y.; Mahadevan, S. 2014. Supplier selection using AHP methodology extended by D numbers, Expert Systems with Applications 41(1): 156-167. https://doi.org/10.1016/j.eswa.2013.07.018

Figueira, J. R.; Greco, S.; Roy, B.; Słowiński, R. 2013. An overview of ELECTRE methods and their recent extensions, Journal of Multi-Criteria Decision Analysis 20(1-2): 61-85. https://doi.org/10.1002/mcda.1482

Garibaldi, J. M.; Ozen, T. 2007. Uncertain fuzzy reasoning: a case study in modelling expert decision making, IEEE Transactions on Fuzzy Systems 15: 16-30. https://doi.org/10.1109/TFUZZ.2006.889755

Grattan-Guiness, I. 1975. Fuzzy membership mapped onto interval and many-valued quantities, Zeitschrift fuer Mathematische Logik und Grundlagen der Mathematik, 22.

Hashemkhani Zolfani, S. H.; Sedaghat, M.; Zavadskas, E. K. 2012. Performance evaluating of rural ICT centers (telecenters), applying fuzzy AHP, SAW-G and TOPSIS Grey, a case study in Iran, Technological and Economic Development of Economy 18(2): 364-387. https://doi.org/10.3846/20294913.2012.685110

Jahn, K. U. 1975. Intervall-wertige Mengen, Mathematische Nachrichten 68: 115-132. https://doi.org/10.1002/mana.19750680109

Kahraman, C.; Cevik Onar, S.; Oztaysi, B. 2015. Fuzzy multicriteria decision-making: a literature review, International Journal of Computational Intelligence Systems 8(4): 637-666. https://doi.org/10.1080/18756891.2015.1046325

Kahraman, C.; Oztaysi, B.; Cevik Onar, S. 2016. A comprehensive literature review of 50 years of fuzzy set theory, International Journal of Computational Intelligence Systems 9(1): 3-24. https://doi.org/10.1080/18756891.2016.1180817

Keshavarz Ghorabaee, M.; Zavadskas, E. K.; Olfat, L.; Turskis, Z. 2015. Multi-criteria inventory classification using a new method of Evaluation Based on Distance from Average Solution (EDAS), Informatica 26(3): 435-451. https://doi.org/10.15388/Informatica.2015.57

Keshavarz Ghorabaee, K.; Zavadskas, E. K.; Amiri, M.; Turskis, Z. 2016. Extended EDAS method for fuzzy multi-criteria decision-making: an application to supplier selection, International Journal of Computers Communications \& Control 11(3): 358-371. https://doi.org/10.15837/ijccc.2016.3.2557

Khan, D.; Samadder, S. R. 2015. A simplified multi-criteria evaluation model for landfill site ranking and selection based on AHP and GIS, Journal of Environmental Engineering and Landscape 23(4): 267-278. https://doi.org/10.3846/16486897.2015.1056741

Kouchaksaraei, R. H.; Hashemkhani Zolfani, S. H.; Golabchi, M. 2015. Glasshouse locating based on SWARA-COPRAS approach, International Journal of Strategic Property Management 19(2): 111-122. https://doi.org/10.3846/1648715X.2015.1004565 
Liu, P. D.; Jin, F.; Zhang, X.; Su, Y.; Wang, M. 2011. Research on the multi-attribute decision-making under risk with interval probability based on prospect theory and the uncertain linguistic variables, Knowledge-Based Systems 24(4): 554-561. https://doi.org/10.1016/j.knosys.2011.01.010

Liu, P. D.; He, L.; Yu, X. C. 2016. Generalized hybrid aggregation operators based on the 2-dimension uncertain linguistic information for multiple attribute group decision making, Group Decision and Negotiation 25(1): 103-126. https://doi.org/10.1007/s10726-015-9434-X

Liu, P. D.; Jin, F. 2012. A multi-attribute group decision-making method based on weighted geometric aggregation operators of interval-valued trapezoidal fuzzy numbers, Applied Mathematical Modelling 36(6): 2498-2509.

https://doi.org/10.1016/j.apm.2011.09.006

Liu, P. D.; Liu, Y. 2014. An approach to multiple attribute group decision making based on intuitionistic trapezoidal fuzzy power generalized aggregation operator, International Journal of Computational Intelligence Systems 7(2): 291-304. https://doi.org/10.1080/18756891.2013.862357

Liu, P. D.; Shi, L. L. 2015. The generalized hybrid weighted average operator based on interval neutrosophic hesitant set and its application to multiple attribute decision making, Neural Computing and Applications 26(2): 457-471. https://doi.org/10.1007/s00521-014-1736-4

Liu, P. D.; Wang, Y. M. 2014. Multiple attribute decision-making method based on single valued neutrosophic normalized weighted Bonferroni mean, Neural Computing and Applications 25(7-8): 2001-2010.

https://doi.org/10.1007/s00521-014-1688-8

Liu, P. D.; Yu, X. C. 2014. 2-dimension uncertain linguistic power generalized weighted aggregation operator and its application for multiple attribute group decision making, KnowledgeBased Systems 57(1): 69-80.

https://doi.org/10.1016/j.knosys.2013.12.009

Liu, P. D.; Zhang, X.; Jin, F. 2012. A multi-attribute group decision-making method based on interval-valued trapezoidal fuzzy numbers hybrid harmonic averaging operators, Journal of Intelligent \& Fuzzy Systems 23(5):159-168.

Mardani, A.; Zavadskas, E. K.; Govindan, K.; Amat Senin, A.; Jusoh, A. 2016. VIKOR technique: a systematic review of the state of the art literature on methodologies and applications, Sustainability 8(1): 37. https://doi.org/10.3390/su8010037

Oztaysi, B.; Cevik Onar, S.; Goztepe, K.; Kahraman, C. 2015. Evaluation of research proposals for grant funding using interval-valued intuitionistic fuzzy sets, Soft Computing 1-16. https://doi.org/10.1007/s00500-015-1853-8

Sambuc, R. 1975. Fonctions $\phi$-floues. Application laide au diagnostic en pathologie thyroidienne: $\mathrm{PhD}$ Thesis. Université Marseille, France.

Seçme, N. Y.; Bayrakdaroğlu, A.; Kahraman, C. 2009. Fuzzy performance evaluation in Turkish banking sector using analytic hierarchy process and TOPSIS, Expert Systems with Applications 36(9): 11699-11709. https://doi.org/10.1016/j.eswa.2009.03.013

Smarandache, F. 1998. Neutrosophy: neutrosophic probability, set, and logic. Rehoboth, USA: American Research Press.
Stanujkic, D.; Magdalinovic, N.; Milanovic, D.; Magdalinovic, S.; Popovic, G. 2014. An efficient and simple multiple criteria model for a grinding circuit selection based on moora method, Informatica 25(1): 73-93.

https://doi.org/10.15388/Informatica.2014.05

Torra, V. 2010. Hesitant fuzzy sets, International Journal of Intelligent Systems 25: 529-539.

https://doi.org/10.1002/int.20418

Turanoglu Bekar, E.; Cakmakci, M.; Kahraman, C. 2016. Fuzzy COPRAS method for performance measurement in total productive maintenance: a comparative analysis, Journal of Business Economics and Management 17(5): 663-684. https://doi.org/10.3846/16111699.2016.1202314

Turskis, Z.; Lazauskas, M.; Zavadskas, E. K. 2012. Fuzzy multiple criteria assessment of construction site alternatives for nonhazardous waste incineration plant in Vilnius city, applying ARAS-F and AHP methods, Journal of Environmental Engineering and Landscape Management 20(2): 110-120. https://doi.org/10.3846/16486897.2011.645827

Turskis, Z.; Zavadskas, E. K.; Kutut, V. 2013. A model based on ARAS-G and AHP methods for multiple criteria prioritizing of heritage value, International Journal of Information Technology \& Decision Making 12(1): 45-73. https://doi.org/10.1142/S021962201350003X

Van Horenbeek, A.; Pintelon, L. 2014. Development of a maintenance performance measurement framework - using the analytic network process (ANP) for maintenance performance indicator selection, Omega 42(1): 33-46. https://doi.org/10.1016/j.omega.2013.02.006

Yager, R. R. 1986. On the theory of bags, International Journal of General Systems 13: 23-37. https://doi.org/10.1080/03081078608934952

Yazdani, M. 2015. New intuitionistic fuzzy approach with multi-objective optimisation on the basis of ratio analysis method, International Journal of Business and Systems Research 9(4): 355-374. https://doi.org/10.1504/IJBSR.2015.072584

Yazdani, M.; Payam, A. F. 2015. A comparative study on material selection of microelectromechanical systems electrostatic actuators using Ashby, VIKOR and TOPSIS, Materials \& Design 65: 328-334.

https://doi.org/10.1016/j.matdes.2014.09.004

Yeh, T. M.; Huang, Y. L. 2014. Factors in determining wind farm location: Integrating GQM, fuzzy DEMATEL, and ANP, Renewable Energy 66: 159-169.

https://doi.org/10.1016/j.renene.2013.12.003

Zadeh, L. A. 1965. Fuzzy sets, Information and Control 8: 338353. https://doi.org/10.1016/S0019-9958(65)90241-X

Zadeh, L. A. 1975. The concept of a linguistic variable and its application to approximate reasoning - 1, Information Sciences 8: 199-249. https://doi.org/10.1016/0020-0255(75)90036-5

Zavadskas, E. K.; Kaklauskas, A.; Turskis, Z.; Kalibatas, D. 2009. An approach to multi-attribute assessment of indoor environment before and after refurbishment of dwellings, Journal of Environmental Engineering and Landscape 17(1): 5-11. https://doi.org/10.3846/1648-6897.2009.17.5-11 
Cengiz KAHRAMAN is a full Professor at Industrial Engineering Department of Istanbul Technical University (ITU). His research areas are engineering economics, quality management, statistical decision making, multiple criteria decision making, and fuzzy decision making. He published about 200 journal papers, about 160 conference papers and 70 book chapters. He is in the editorial boards of 20 international journals and edited 10 international books from Springer. He guest-edited many special issues of international journals and organized international conferences. He was the vice dean of ITU Management Faculty between the years 2004-2007 and the head of ITU Industrial Engineering Department between the years 2010-2013.

Mehdi KESHAVARZ GHORABAEE received the BS degree in electrical engineering from the University of Guilan, Rasht, Iran in 2010 and the MS degree in production management from the Allame Tabataba'i University, Tehran, Iran in 2013. $\mathrm{He}$ is currently working toward the $\mathrm{PhD}$ degree in management (Operations Research) at Allame Tabataba'i University. He has published some papers in leading international journals. His research interests include multi-criteria decision making (MCDM), multi-objective evolutionary algorithms, genetic algorithm, fuzzy MCDM, inventory control, supply chain management, scheduling and reliability engineering.

Edmundas Kazimieras ZAVADSKAS is a full professor and the Head of the Department of Construction Technology and Management of Vilnius Gediminas Technical University, Lithuania. Senior Research Fellow at the Research Institute of Smart Building Technologies. PhD in Building Structures (1973). Dr Sc. (1987) in Building Technology and Management. A member of Lithuanian and several foreign Academies of Sciences. Doctore Honoris Causa from Poznan, Saint Petersburg and Kiev universities. The Honorary International Chair Professor in the National Taipei University of Technology. A member of international organizations; a member of steering and programme committees at many international conferences; a member of the editorial boards of several research journals; the author and co-author of more than 400 papers and a number of monographs in Lithuanian, English, German and Russian. Editor-in-chief of journals Technological and Economic Development of Economy and Journal of Civil Engineering and Management. Research interests: building technology and management, decision-making theory, automation in design and decision support systems.

Sezi CEVIK ONAR is a full time Associate Professor at Industrial Engineering Department of Istanbul Technical University (ITU). Her research interests include multiple criteria decision making, supply chain management and fuzzy systems. She has published 15 articles, 35 conference papers and 8 book chapters. She has edited a book on Engineering Management which is published by Springer. Dr Cevik Onar teaches courses on human resources management, business planning, management and organization.

Morteza YAZDANI is a PhD student in business and economics in Faculty of Business and Communication at Universidad Europea de Madrid, Spain. His main research area is the application of multi criteria decision making in supply chain management, sustainability and strategic planning. He has published papers in some international journals such as International Journal of Logistics Research, International Journal of Strategic Decision Science, Expert Systems with Applications, Engineering Economics, Journal of Civil Engineering and Management, Technological and Economic Development of Economy and Materials \& Design.

Basar OZTAYSI is a full time Associate Professor at Industrial Engineering Department of Istanbul Technical University (ITU). His research interests include multiple criteria decision making, data mining and intelligent systems. He has published 25 articles, 32 conference papers and 10 book chapters. He has edited a book on Supply Chain Management which is published by Springer. Başar Öztayşi teaches courses on data management, information systems management and business intelligence and decision support systems. 